\section{Propiedades Psicométricas del Perfil y el Inventario de Personalidad de Gordon "P-IPG" en una muestra de adultos de la ciudad de Bogotáio}

\author{
Carlos Eduardo González Cifuentes \\ Magister en Psicología Clínica \\ Universidad Cooperativa de Colombia \\ Bogotá \\ Correo electrónico: carlose.gonzalezc@gmail.com
}

Fernando Riveros

Magister en Psicología Universidad de La Sabana Chía, Colombia Correo electrónico: efriveros45@hotmail.com

Orfidea Ovalle Garzón

Magister en Informática Educativa Universidad Cooperativa de Colombia Bogotá Correo electrónico: ovgarzono@gmail.com

Luis Anderssen Vera Maldonado

Magister en Educación Universidad de San Buenaventura Bogotá

Correo electrónico: Ivera@usbbog.edu.co
Recibido: 17/04/2017 Evaluado: 09/07/2018 Aceptado: 26/07/2018

\title{
Resumen
}

El objetivo de la presente investigación de tipo instrumental fue explorar la validez factorial, la confiabilidad y otras propiedades psicométricas de la prueba de personalidad P-IPG de Gordon (1972; 1994) en una muestra de la ciudad de Bogotá - Colombia. Para tal fin se contó con la participación de 600 hombres y mujeres mayores de 18 años residentes en el área metropolitana de la ciudad de Bogotá a quienes se les aplicaron las escalas. Los resultados indican que la sub-prueba del PPG mostró ajustarse a un comportamiento tetrafactorial que validó la existencia de factores denominados Ascendencia, Responsabilidad, Estabilidad y Sociabilidad. La sub-prueba IPG arrojó una solución pentafactorial donde emergieron los factores propuestos por Gordon de Cautela, Originalidad, Relaciones Personales, Vigor y un nuevo factor denominado Autocontrol Emocional. Los coeficientes de fiabilidad alfa de Cronbach de las escalas del P- IPG oscilaron entre .60 y .72. En conclusión, puede afirmarse que la prueba P-IPG es válida y confiable para su uso en el contexto colombiano.

\section{Palabras clave}

Personalidad, Inventario de Personalidad, Validez, Confiabilidad, Propiedades Psicométricas.

12 Para citar este artículo: González, C.E., Riveros, F., Ovalle, O. \& Vera, A. (2019). Propiedades Psicométricas del Perfil y el Inventario de Personalidad de Gordon "P-IPG" en una muestra de adultos de la ciudad de Bogotá. Informes Psicológicos, 19(1), pp. 181-196 http://dx.doi.org/10.18566/infpsic.v19n1a010 


\section{Psychometric properties of the profile and the Gordon Personality Inventory "P-IPG" in a sample of adults in the city of Bogotá}

Abstract

The objective of this instrumental research was to explore the factorial validity, reliability and other psychometric properties of the GPP-I, Gordon Personal Profile Inventory $(1972 ; 1994)$ in a sample in the city of Bogotá - Colombia. For this purpose, there were 600 men and women over 18 years of age living in the metropolitan area of the city of Bogotá to whom the scales were applied. The results indicate that the PPG subtest adjusted to a tetrafactorial behavior that validated the existence of factors such as Ascendancy, Responsibility, Stability and Sociability. The IPG sub-test yielded a pentafactorial solution where the factors of Stealth, Originality, Personal Relationships, Vigor and a new factor called Emotional Self-Control proposed by Gordon emerged. The Cronbach's alpha reliability coefficients of the P-IPG scales ranged between .60 and .72. In conclusion, it can be affirmed that the P-IPG test is valid and reliable for its use in the Colombian context.

Keywords

Personality, personality inventory, validity, reliability, psychometric properties.

\section{Propriedades Psicométricas do Perfil e 0} Inventário de Personalidade Gordon "P-IPG" em uma amostra de adultos da cidade de Bogotá

Resumo

0 objetivo desta pesquisa instrumental foi explorar a validade fatorial, a confiabilidade e outras propriedades psicométricas do teste de personalidade P-IPG de Gordon (1972; 1994) em uma amostra da cidade de Bogotá - Colômbia. Para este fim, tomaram parte 600 homens e mulheres com mais de 18 anos residentes na região metropolitana da cidade de Bogotá, aos quais foram aplicadas as escalas. Os resultados indicam que o subteste do PPG se mostrou adequado a um comportamento tetrafatorial que validou a existência de fatores denominados Ascendência, Responsabilidade, Estabilidade e Sociabilidade. 0 sub-teste IPG rendeu uma solução pentafatorial onde surgiram os fatores propostos por Gordon de Stealth, Originalidade, Relações Pessoais, Vigor e um novo fator chamado Autocontrole Emocional. Os coeficientes de confiabilidade alfa de Cronbach das escalas do P-IPG variaram entre .60 e .72. Em conclusão, pode-se afirmar que o teste P-IPG é válido e confiável para seu uso no contexto colombiano.

Palavras chave

Personalidade, inventário de personalidade, validade, confiabilidade, propriedades psicométricas 


\section{ntroducción}

La apropiada validación y estandarización de instrumentos y pruebas psicológicas en Colombia es una tarea que está en mora de realizarse a pesar de los ya más de 50 años de la psicología colombiana, en tanto que la mayoría de instrumentos psicométricos que se usan a nivel nacional tengan que utilizarse con las adaptaciones y baremaciones extranjeras de otros países de habla castellana como España, Argentina y México; el campo de la evaluación y medición psicológica en Colombia permanecerá en un rezago profesional e investigativo. Sobre este aspecto, González (2009) ha indicado que una de las dificultades principales con la que se encuentra cualquier investigador del área social en Colombia es precisamente la falta de instrumentos adecuadamente validados y estandarizados para este contexto particular, por lo que es necesario impulsar y divulgar la investigación en medición y evaluación a nivel regional y local, ya que el uso de escalas sin datos de validez y confiabilidad enfrenta serias amenazas a los diversos tipos de validez.

Sobre ello, es sabido por la comunidad científica que las pruebas psicológicas no poseen características intrínsecamente universales (Anastasi \& Urbina, 1998) y que los estudios de análisis del comportamiento de la prueba con poblaciones específicas son siempre necesarios, además de ser una práctica éticamente responsable. Sobre lo anterior, Kerlinger y Lee (2002) refieren que el adecuado proceso de validación y obtención de normas, poblaciones y baremos debe realizarse para cada contexto étnico y cultural determinado en el cual se utilizará la prueba con el fin de obtener resultados válidos y confiables en las aplicaciones individuales, asunto que recae especialmente en instrumentos de uso clínico tal como las escalas de personalidad, específicamente al Perfil e Inventario de Personalidad de Gordon.

El Perfil e Inventario de Personalidad de Gordon (P-IPG; Gordon, 1972; 1994) es una escala conformada por dos instrumentos diferentes, el perfil de personalidad y el inventario de personalidad, ambos derivados desde la teoría de rasgos, los cuales son entendidos como patrones constantes en la forma en que un individuo siente, piensa y se comporta (Cervone \& Pervin, 2009); los rasgos son conceptualizados como la unidad descriptiva de la personalidad, son constructos predisposicionales que son estables en el tiempo, transituacionales y distintivos en el comportamiento de un individuo (McCrae \& Costa, 1999). Tras el análisis en conjunto con las dos escalas se observa una apropiada funcionalidad, por lo que se toma la decisión de vincularlos en un único instrumento. Cuenta desde sus inicios con sólidos estudios de sus propiedades psicométricas y su aplicación es susceptible en las principales áreas de la psicología. Sin embargo, en Colombia está disponible la traducción y la adaptación mexicana, de la cual se desconoce su ajuste al contexto nacional, sin datos del comportamiento psicométrico de la misma. Aunque se trata de una prueba de personalidad robusta psicométricamente, según los estudios originales de confiabilidad y la validez (Gordon, 1972; 1994), su popularidad parece haber decrecido en los últimos años muy probablemente debido a la carencia de datos psicométricos actualizados con población colombiana. 
Aun cuando no existe ningún estudio de validación de la prueba en Colombia, los análisis realizados en otros contextos refieren un análisis factorial de la prueba bastante consistente a lo largo de una serie de estudios en diversos países como Estados Unidos e India (Gordon, 1972; 1994). En bases de datos especializadas no se encuentra reporte en los últimos cinco años de estudios de validación del instrumento en inglés o en español, sin embargo, este fenómeno puede deberse que muchos países cuentan con sus propias adaptaciones hace ya un tiempo considerable. Los estudios más cercanos a Colombia, son los realizados por Martínez, Romero y Trejo (1994), quienes hicieron el proceso de validación para México.

Sobre algunas aplicaciones de la escala se encuentra un estudio que analizó los rasgos de personalidad con mayor incidencia en una población mexicana con obesidad (Bravo del Toro, Espinosa, Mancilla \& Tello, 2011), donde se contó con una muestra de 60 pacientes, distribuidos en 31 hombres y 29 mujeres, con edades de 30 a 40 años, casados, con nivel educativo básico y que acudían a consulta médica en un centro de diagnóstico clínico, con antecedentes de obesidad, hallando que los pacientes con obesidad tienden a mostrar rasgos de personalidad por debajo de la norma, mostrando además relaciones significativas entre la gente con obesidad y cada uno de los rasgos de personalidad con menor prevalencia que mide la escala.

Otro estudio buscó el perfil psicosocial y uso de las tecnologías de la información y la comunicación de alumnos con promedios académicos altos y mínimos de la modalidad educativa presencial en un contexto mexicano (Rodríguez,
Ávila, González \& Heredia, 2008), encontrando una diferencia estadística significativa a favor de los alumnos encuestados con promedio académico alto, para las áreas cognoscitiva, emocional, uso de las TIC y (específicamente en personalidad) en autoestima. Finalmente, un estudio colombiano que utilizó el P-IPG (Sierra, Barrera, Gómez, Jiménez \& Vélez, 2008) buscó identificar cuáles son las competencias comunes en un grupo de docentes de una facultad de psicología en la ciudad de Bogotá, encontrando que la muestra de estudio se encuentra motivada hacia el salario, en cuanto a los aspectos de personalidad, puntuaciones altas en ascendencia, autoestima y relaciones personales, y baja puntuación en sociabilidad.

En suma, existe evidencia de la utilización de la Prueba de Personalidad P-IPG a nivel internacional y a nivel local, y se cuenta con datos de validez y confiabilidad en otros países más no en Colombia, por lo que el objetivo de la presente investigación fue explorar la validez factorial, la confiabilidad y otras propiedades psicométricas de la prueba de personalidad P-IPG de Gordon (1972; 1994) en una muestra de adultos de la ciudad de Bogotá.

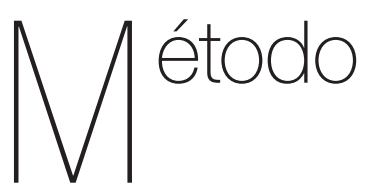

\section{Tipo de investigación}

Según la clasificación de Montero y León (2007), el presente es un estudio de corte transversal, de alcance descriptivo y de tipo instrumental. 


\section{Participantes}

En este estudio participaron 600 personas de ambos sexos (52.3\% hombres y $47.7 \%$ mujeres) pertenecientes a la ciudad de Bogotá y su área metropolitana, con edades comprendidas entre los 18 y los 67 años de edad (M $=29.9$ y DE $=8.8$ ). En la muestra las ocupaciones actuales de los participantes fueron: estudiante de bachillerato (1.2\%), estudiante de educación superior (19.7\%), empleado del sector público $(6 \%)$, empleado del sector privado (33.2\%), independiente (6.2\%), vendedor $(2 \%)$, hogar (3.5\%), militar (14.7\%), desempleado (13.3\%) y otra ocupación (.3\%). Según su estrato socioeconómico, se distribuyeron como: $39.98 \%$ bajo; $58.3 \%$ medio; $1.8 \%$ alto y .5\% sin estrato. Se realizó un muestro no probabilístico mediante una estrategia de bola de nueve (Hernández, Fernández \& Baptista, 2014).

\section{Instrumentos}

Se utilizó el Perfil e Inventario de Personalidad de Gordon (P-IPG; Gordon, 1972; 1994) compuesto por la compilación de dos pruebas de personalidad construidas inicialmente de manera independiente y compiladas en una edición única dadas sus características complementarias en cuanto a los rasgos medidos. La prueba total consta de 34 reactivos presentados en tétradas de elección forzada, donde debe seleccionarse una frase que se acomode al participante y una más que vaya en contra de las características del mismo participante; los primeros 18 reactivos corresponden al Perfil de Personalidad de Gordon (PPG) y conforman cuatro subescalas: ascendencia, responsabilidad, estabilidad y sociabilidad; los reactivos restantes corresponden al Inventario de Personalidad de Gordon (IPG), segmentadas en las subescalas de cautela, originalidad, relaciones personales y vigor. El rango de coeficientes alfa de Cronbach para el PPG se encuentra entre .85 a .88 y para el IPG entre .83 y .87 según el manual de la prueba. Para esta investigación se contó con la versión adaptada a México por Martínez et al. (1994).

\section{Procedimiento}

Tras el proceso de capacitación al equipo de auxiliares de investigación (dominio de las condiciones de aplicación, de la escala y su calificación, así como de la elaboración de la base de datos), se procedió con una prueba piloto para verificar las condiciones semánticas, de lenguaje y cultural de la misma, seguido de la consecución de la muestra global, la firma de consentimientos informados y aplicación de los instrumentos a los participantes. Los análisis de resultados se realizaron por medio del paquete estadístico SPSS versión 22 (IBM, 2013).

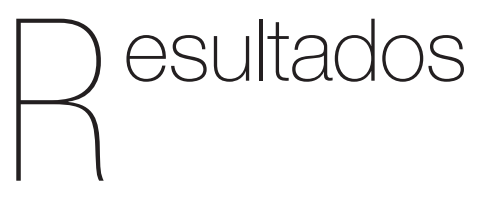

Con el fin de evaluar la adecuación semántica y cultural de los reactivos al contexto colombiano se realizó el pilotaje cualitativito para el P-IPG, mostrando que, a pesar que el formato de respuesta de elección forzada usado en la prueba genera resistencia al inicio, las 
personas se adaptan a él, y que algunas de las personas que realizaron la prueba tuvieron dificultades con los ítems negativos requiriendo aclaraciones. En términos generales, se encontró que los reactivos contenidos en el instrumento P-IPG se presentan en un castellano neutro, se entienden bien y no presentan barreras lingüísticas, semánticas o pragmáticas.

Como se mencionó en la introducción, la prueba del Perfil e Inventario de Personalidad de Gordon (P-IPG, 1972; 1994) está compuesta por dos pruebas desarrolladas de manera independiente que, gracias a que usan el mismo formato de reactivo en tétradas, fueron compiladas en la versión comercial para lograr en una sola aplicación mayor amplitud en la evaluación de la personalidad. EI PPG pretende medir cinco escalas y el IPG pretende medir cuatro escalas; por tanto, los dos instrumentos en conjunto logran evaluar un total de nueve escalas de rasgos personalidad. Así las cosas, para el proceso de evaluación de la validez del P-IPG en realidad deben realizarse dos análisis simultáneos pero independientes: uno para el Perfil de Personalidad de Gordon PPG y otro para el Inventario de Personalidad de Gordon IPG. En este orden de ideas, el plan de análisis de datos desarrollado para la presente investigación contempló la conducción y reporte de resultados para cada prueba por separado siendo el primer análisis para el PPG y el segundo para el IPG.

\section{Análisis del PPG}

En el caso de los reactivos que componen el PPG se analizaron las tétradas de la 1 hasta 18. Con el fin de determinar los coeficientes de confiabilidad, se procedió a obtener los alfas de Cronbach con los reactivos que conformaban cada subescala del PPG según el manual de la prueba, encontrándose alfas medios para las escalas; se atribuyen estos resultados a un posible comportamiento factorial diferente al descrito por el manual (correspondiente a población estadounidense) y/o a otros factores inespecíficos. Los coeficientes de fiabilidad y otros estadísticos descriptivos de las escalas completas se presentan en la Tabla 1

Tabla 1

Coeficientes Alfa de Cronbach de las Escalas Completas del PPG para la muestra total.

\begin{tabular}{lccc}
\hline \multicolumn{1}{c}{ Subescala } & $\alpha$ & M & DS \\
\hline Ascendencia & 0.69 & 23.25 & 4.38 \\
Responsabilidad & 0.72 & 26.14 & 4.39 \\
Estabilidad & 0.68 & 25.11 & 4.27 \\
Sociabilidad & 0.63 & 22.64 & 3.79 \\
\hline
\end{tabular}

El siguiente paso lógico del análisis de datos es la realización de análisis factoriales de las 18 tétradas de reactivos que constituyen el PPG: siguiendo los estudios referenciados por el manual se procedió a solicitar cuatro factores fijos mediante el método de componentes principales con rotación Varimax (debido a la ortogonalidad de los datos). Se realizó un análisis factorial ingresando únicamente los reactivos presentados por el manual de la prueba como solución factorial de las escalas del PPG intentando replicar la estructura factorial. Se encontró una adecuación muestral KMO de .50; una prueba de esfericidad de Barlett significativa al .01 y una varianza total explicada de $34.6 \%$ en cuatro factores. Los resultados de la estructura factorial se presentan en la Tabla 2. 
Tabla 2

Estructura Factorial Original PPG

\begin{tabular}{|c|c|c|c|c|}
\hline & Sociabilidad & Estabilidad & Responsabilidad & Ascendencia \\
\hline & 1 & 2 & 3 & 4 \\
\hline A2R no le interesa estar con otras personas & 0.525 & 0.003 & 0.129 & 0.043 \\
\hline C8R limita sus relaciones sociales a unos cuantos & 0.519 & -0.353 & -0.004 & -0.14 \\
\hline A4R se le facilita hacer nuevas amistades & -0.497 & 0.094 & 0.133 & -0.18 \\
\hline C16R disfruta rodeándose de mucha gente & -0.478 & -0.436 & 0.031 & 0.232 \\
\hline A1R Es bastante sociable & -0.469 & 0.005 & 0.39 & -0.295 \\
\hline A8R le resulta fácil influir en los demás & -0.414 & -0.169 & 0.317 & -0.249 \\
\hline B15R no le interesa unirse a actividades grupales & 0.408 & -0.076 & 0.186 & -0.176 \\
\hline D17R toma parte activa en las discusiones de grupo & -0.37 & -0.342 & -0.046 & -0.153 \\
\hline B16R no puede perseverar en el trabajo que realiza & 0.351 & 0.075 & 0.287 & 0.041 \\
\hline B14R Prefieren que otros dirijan las actividades de grupo & 0.304 & -0.03 & -0.183 & -0.287 \\
\hline C7R no se siente muy seguro(a) de sus propias opiniones & 0.092 & 0.704 & 0.095 & -0.078 \\
\hline B7R se disgusta facialmente cuando las cosas van mal & 0.153 & -0.651 & 0.076 & 0.2 \\
\hline D8R tiende a ser una persona más bien nerviosa & -0.074 & 0.531 & 0.092 & 0.344 \\
\hline C2R es una persona poco confiable & -0.148 & 0.33 & 0.11 & 0.191 \\
\hline C18R carece de seguridad en sí mismo(a) & 0.041 & 0.326 & 0.216 & 0.178 \\
\hline B13R le falta sentido de responsabilidad & 0.237 & 0.133 & 0.608 & 0.082 \\
\hline D3R es un(a) trabajador(a) muy persistente y formal & -0.161 & 0.116 & -0.569 & 0.036 \\
\hline C1R es perfeccionista con cualquier trabajo que realiza & 0.215 & -0.094 & -0.555 & 0.1 \\
\hline A13R se siete libre de inquietudes y preocupaciones & 0.059 & -0.161 & -0.481 & -0.097 \\
\hline A5R es capaz de tomar decisiones importantes sin ayuda & -0.158 & -0.035 & 0.043 & -0.707 \\
\hline D5R concluye su trabajo a pesar de los problemas & -0.095 & -0.069 & -0.272 & 0.581 \\
\hline C14R parece estar siempre preocupado(a) & 0.024 & 0.136 & 0.365 & 0.424 \\
\hline C17R le resulta difícil relajarse & 0.068 & 0.053 & 0.057 & 0.381 \\
\hline A16R es calmado(a) y fácil de tratar & 0.155 & 0.321 & -0.325 & -0.362 \\
\hline Replicación del Factor & $100 \%$ & $33.33 \%$ & $50 \%$ & $16.66 \%$ \\
\hline
\end{tabular}

Nota: Método de extracción: análisis de componentes principales y Método de rotación: Varimax: $n=600$

Acogiendo la indicación de Gordon (1994) de utilizar como criterio de carga factorial valores de .40 para identificar los factores, puede aislarse claramente un primer factor que se denominó por su contenido semántico como sociabilidad; este factor es idéntico al referido por el autor de la prueba en el manual, sin embargo, los otros factores no resultaron ser tan definidos como el primero. El segundo factor que puede aislarse según los datos de la tabla es un factor que por su contenido semántico se denominó estabilidad emocional, pero éste sólo está conformado por tres reactivos y no replica el factor original. El tercer factor se denominó por análisis semántico responsabilidad, está conformado por cuatro reactivos, tres del factor de responsabilidad original y otro proveniente del factor de estabilidad; este 
tercer factor replica en un 50\% al factor original. El cuarto factor está constituido por tres reactivos y se denominó ascendencia definido por el reactivo con carga factorial más alta, empero este factor no replica el factor original de la prueba.

Dado que la estructura factorial de las escalas propuesta por el manual del instrumento no pudo ser replicada de manera satisfactoria en la muestra colombiana y que los coeficientes alfa de Cronbach de las escalas se encuentran por debajo de los estándares recomendados en la comunidad científica, la matriz de correlaciones no resulta pertinente para el análisis replicando los hallazgos originales de la escala, se condujeron análisis factoriales de la prueba conservando los cuatro factores fijos ya reportados en los estudios originales de construcción (Gordon, 1972; 1994) pero ingresando la totalidad de reactivos. Se realizaron múltiples versiones de estructuras factoriales hasta encontrar aquella combinación de reactivos y solución factorial que lograse equilibrar aceptables resultados en la varianza total explicada, los coeficientes alfas de las escalas resultantes y un $\mathrm{KMO}$ admisible. El criterio de carga de factorial para identificar los factores extraídos se conservó en .40 según el manual, aquellos reactivos con cargas inferiores (o con cargas en más de un factor) fueron eliminados de la solución final. La solución factorial para la muestra colombiana se presenta en la Tabla 3.

Tabla 3

Solución Factorial PPG en la muestra de Bogotá - Colombia

\begin{tabular}{|c|c|c|c|c|}
\hline & Estabilidad & Ascendencia & Responsabilidad & Sociabilidad \\
\hline & 1 & 2 & 3 & 4 \\
\hline C15R es una persona muy nerviosa & -0.594 & & & \\
\hline B10R sus sentimientos son heridos fácilmente & -0.558 & & & \\
\hline D8R tiende a ser una persona más bien nerviosa & -0.546 & & & \\
\hline A3R actúa de manera nerviosa e inestable & -0.525 & & & \\
\hline C5R tiende a sentirse tenso(a) o muy presionado(a) & -0.496 & & & \\
\hline D9R no se encuentra emocionalmente equilibrado (a) & -0.481 & & & \\
\hline A18R no se deja vencer fácilmente por un problema & 0.467 & & & \\
\hline C2R es una persona poco confiable & -0.423 & & & \\
\hline D17R toma parte activa en las discusiones de grupo & & 0.72 & & \\
\hline A17R es una persona totalmente confiable & & -0.591 & & \\
\hline D6R toma el mando en actividades de grupo & & 0.568 & & \\
\hline D2R toma la conducción en las discusiones de grupo & & 0.521 & & \\
\hline D7R prefiere estar cerca de la gente & & 0.521 & & \\
\hline B9R toma parte activa en los asuntos de grupos & & 0.516 & & \\
\hline D12R tiende a ser muy sociable & & 0.514 & & \\
\hline C16R disfruta rodeándose de mucha gente & & 0.472 & & \\
\hline D15R es muy persistente en el trabajo que realiza & & & 0.718 & \\
\hline B8R termina su trabajo a pesar de los obstáculos & & & 0.674 & \\
\hline
\end{tabular}




\begin{tabular}{|c|c|c|c|c|}
\hline & Estabilidad & Ascendencia & Responsabilidad & Sociabilidad \\
\hline & 1 & 2 & 3 & 4 \\
\hline A15R es capaz de cambiar las opiniones de otros & & & -0.66 & \\
\hline A8R le resulta fácil influir en los demás & & & -0.573 & \\
\hline D3R es un(a) trabajador(a) muy persistente y formal & & & 0.48 & \\
\hline D11R es perfeccionista en el trabajo que realiza & & & 0.464 & \\
\hline A14R Le resulta fácil ser amistoso(a) & & & & 0.712 \\
\hline A4R se le facilita hacer nuevas amistades & & & & 0.584 \\
\hline D14R persevera en un trabajo a pesar de los problemas & & & & -0.533 \\
\hline A1R Es bastante sociable & & & & 0.521 \\
\hline A10R se siente seguro(a) en sus relaciones con los demás & & & & 0.499 \\
\hline A9R no hace amigos fácilmente & & & & -0.464 \\
\hline A6R no le interesa mucho ser sociable & & & & -0.405 \\
\hline
\end{tabular}

Nota: $n=600$

La solución factorial en la muestra arrojó una medida KMO de .75 con una prueba de esfericidad de Barlett significativa al .01 indicando que la solución es admisible. La varianza total explicada del análisis factorial es de $37.12 \%$. Se conservaron los cuatro factores fijos reportados por el autor y se conservó como criterio la carga factorial de .40 para identificar los factores. La estructura tetrafactorial resultante agrupa los ítems de manera adecuada a nivel semántico. El primer factor explica el $10 \%$ de la varianza y se denominó estabilidad emocional; el segundo factor explica el 9.57 \% y se denominó ascendencia; el tercer factor explica $8.79 \%$ de la varianza y se denominó responsabilidad; el cuarto factor explica el $8.76 \%$ de la varianza y se denominó sociabilidad.

Partiendo de la solución factorial se procedió al cálculo de los coeficientes de fiabilidad y estadísticos descriptivos de las nuevas escalas revisadas. Para las cuatro escalas, los coeficientes alfa de Cronbach oscilan entre .64 y .69 que estarían por encima del mínimo aceptable tradicional de .60 (ver Tabla 4). Estos valores indican que la intercorrelación promedio entre los reactivos de cada escala es moderada (Brown, 1980). Según otros autores como George y Mallery (2003), los coeficientes dentro del rango de .60 a .69 son cuestionables y los coeficientes aceptables suelen encontrarse por encima de .70 (Campos \& Oviedo, 2008).

Tabla 4

Coeficientes Alfa de Cronbach de las Escalas Revisadas

\begin{tabular}{lcccc}
\hline \multicolumn{1}{c}{ Escala } & Elementos & $\alpha$ & M & DS \\
\hline Estabilidad & 8 & .65 & 4.08 & 2.59 \\
Ascendencia & 8 & .69 & 8.54 & 2.73 \\
Responsabilidad & 6 & .67 & 7.69 & 2.18 \\
Sociabilidad & 7 & .64 & 8.66 & 2.46 \\
\hline
\end{tabular}

\section{Análisis del IPG}

En el caso de los reactivos que componen el IPG se analizaron las tétradas de la 19 hasta la 38. Al igual que con el PPG, inicialmente se realizó un análisis de confiabilidad de las escalas según el manual de la prueba, encontrándose coeficientes de fiabilidad cuestionables para las cuatro 
escalas según la clasificación de George y Mallery (2003). Los resultados son presentados en la Tabla 5.

Tabla 5

Coeficientes Alfa de Cronbach de las Escalas Completas del PPG

\begin{tabular}{lccc}
\hline \multicolumn{1}{c}{ Escala } & Alfa & M & DT \\
\hline Cautela & .64 & 24.06 & 4.64 \\
Originalidad & .60 & 25.20 & 3.98 \\
R. Personales & .68 & 24.42 & 4.93 \\
Vigor & .64 & 26.46 & 4.37 \\
\hline
\end{tabular}

De igual forma, se llevó a cabo el análisis factoriales de las 20 tétradas de reactivos que constituyen el IPG; siguiendo los estudios referenciados por el manual se procedió a solicitar cuatro factores fijos mediante el método de componentes principales con rotación Varimax. Se realizó un análisis factorial ingresando únicamente los reactivos presentados por el manual de la prueba como solución factorial original de las escalas del IPG intentando replicar la estructura factorial. Se encontró una adecuación muestral $\mathrm{KMO}$ de .45, una prueba de esfericidad de Barlett significativa al .01 y una varianza total explicada de $31.62 \%$ en cuatro factores. Los resultados de la estructura factorial se presentan en la Tabla 6.

Tabla 6

Estructura Factorial Original IPG

\begin{tabular}{|c|c|c|c|c|}
\hline & Indefinido & Indefinido & Indefinido & Indefinido \\
\hline & 1 & 2 & 3 & 4 \\
\hline A20R cree que toda la gente es esencialmente honesta & -.774 & -.137 & .027 & -.077 \\
\hline C36R se ofende cuando es criticado(a) & .514 & -.187 & .238 & -.187 \\
\hline D20R tiende a actuar impulsivamente & .507 & -.276 & .372 & -.105 \\
\hline B32R tiene mucha fe en la gente & -.47 & .05 & .182 & -.058 \\
\hline A36R puede realizar más cosas que otras personas & -.429 & .063 & -.087 & -.036 \\
\hline A32R no le gusta trabajar rápidamente & .402 & .007 & -.201 & -.217 \\
\hline A30R pierde la paciencia con los demás rápidamente & .175 & -.741 & -.048 & -.153 \\
\hline D24R emplea bastante tiempo pensando en nuevas ideas & .062 & .572 & -.125 & -.096 \\
\hline B20R le gusta tomar con calma el trabajo o el juego & .306 & .492 & -.353 & .164 \\
\hline B24R se irrita fácilmente con los demás & .276 & -.418 & .244 & -.377 \\
\hline D30R no le interesa mucho lo emocionante & -.093 & .395 & .073 & -.074 \\
\hline B25R busca lo emocionante y excitante & .166 & -.376 & .035 & .251 \\
\hline B30R tiene menos resistencia que la mayoría de la gente & .039 & .324 & .231 & -.146 \\
\hline B34R es muy cauteloso(a) antes de actuar & -.025 & -.008 & -.764 & .06 \\
\hline A34R no habla sino lo mejor de otras personas & -.134 & .043 & .54 & .065 \\
\hline B35R no actúa impulsivamente & -.113 & .13 & -.426 & .018 \\
\hline A35R no tiene una mente inquisitiva & .03 & .156 & .207 & -.057 \\
\hline A19R tiene ideas muy originales & -.04 & .104 & .18 & .603 \\
\hline A33R es un (a) trabajador (a) muy activo (a) & .009 & -.058 & -.026 & .493 \\
\hline A24R se distingue por arriesgarse & -.17 & -.185 & -.049 & .448 \\
\hline A23R le gusta trabajar principalmente con ideas & .005 & .111 & .367 & .42 \\
\hline D25R prefiere poner en práctica un proyecto que planearlo & .032 & .04 & .168 & -.408 \\
\hline C38R mantiene un ritmo vivaz en el trabajo o el juego & .134 & .117 & -.267 & .293 \\
\hline C27R carece de interés para pensar de manera critica & -.028 & .078 & .027 & -.266 \\
\hline
\end{tabular}

Nota: $\mathrm{n}=600$ 
La estructura factorial original del IPG presentada en el manual de la prueba no pudo ser replicada en este estudio ya que el KMO es inadecuado y los cuatro factores son indefinidos, es decir, los factores resultantes en realidad contienen ítems correspondientes a todas las escalas de la prueba sin mostrar un claro agrupamiento semántico concordante con las escalas de la prueba propuestas por Gordon (1972; 1994) razón por la cual esta solución factorial se considera inadecuada para la muestra en cuestión (Pérez, 2005) .

Al igual que para el análisis del PPG, se procedió a realizar análisis factoriales con el fin de encontrar la mejor solución factorial de la prueba IPG en la muestra encontrándose que los datos no parecen ajustarse a una solución de cuatro factores o tres factores, ya que se obtenían factores indefinidos para tres de las cuatro escalas de la prueba. Dado que el gráfico de saturación según el punto de inflexión sugirió cinco factores (Hernández \& González, 2006; Peña, 2002), resultado también hallado en el análisis paralelo de Horn, se realizó una solución con cinco factores obteniendo una mejor definición semántica de las escalas de la prueba y cuyos resultados se muestran en la Tabla 7.

Tabla 7

Solución Factorial IPG en la muestra de Bogotá - Colombia

\begin{tabular}{|c|c|c|c|c|c|}
\hline & $\begin{array}{c}\text { Auto- } \\
\text { control } \\
\text { Emocional }\end{array}$ & Vigor & $\begin{array}{c}\text { R. } \\
\text { Personales }\end{array}$ & Cautela & $\begin{array}{l}\text { Origina- } \\
\text { lidad }\end{array}$ \\
\hline & 1 & 2 & 3 & 4 & 5 \\
\hline D35R se irrita fácilmente por las debilidades de los demás| & -0.588 & -0.104 & -0.133 & -0.102 & -0.129 \\
\hline A30R pierde la paciencia con los demás rápidamente & -0.572 & -0.183 & -0.008 & 0.136 & -0.025 \\
\hline C30R tiende a ser creativo(a) y original & 0.561 & 0.307 & -0.11 & -0.04 & 0.188 \\
\hline A25R es una persona muy paciente & 0.549 & -0.182 & -0.041 & 0.001 & -0.378 \\
\hline B35R no actúa impulsivamente & 0.536 & -0.35 & 0.039 & 0.176 & -0.008 \\
\hline B24R se irrita fácilmente con los demás & -0.524 & -0.253 & -0.112 & -0.123 & -0.202 \\
\hline B27R se irrita con los errores de los demás & -0.491 & -0.103 & -0.122 & -0.116 & -0.118 \\
\hline D20R tiende a actuar impulsivamente & -0.484 & -0.095 & -0.274 & -0.08 & -0.128 \\
\hline A27R no actúa impulsivamente & 0.467 & -0.168 & 0.037 & 0.099 & 0.11 \\
\hline A28R tiende a disgustarse mucho con la gente & -0.456 & -0.193 & -0.061 & -0.002 & 0.035 \\
\hline B20R le gusta tomar con calma el trabajo o el juego & 0.432 & -0.05 & -0.362 & 0.102 & -0.104 \\
\hline C35R generalmente esta desbordante de energía & -0.076 & 0.639 & 0.068 & -0.093 & 0.124 \\
\hline B31R tiene un gran vigor y dinamismo & 0.088 & 0.594 & 0.194 & 0.045 & -0.065 \\
\hline B28R le gusta estar siempre activo(a) & 0.309 & 0.49 & 0.083 & 0.01 & 0.061 \\
\hline A21R es una persona muy activa & 0.138 & 0.451 & -0.191 & 0.036 & 0.097 \\
\hline A33R es un (a) trabajador (a) muy activo (a) & 0.034 & 0.45 & -0.091 & -0.03 & 0.088 \\
\hline A37R confía mucho en las personas & 0.135 & -0.217 & 0.665 & 0.076 & -0.037 \\
\hline
\end{tabular}




\begin{tabular}{lccccc}
\hline & $\begin{array}{c}\text { Auto- } \\
\text { control } \\
\text { Emocional }\end{array}$ & Vigor & $\begin{array}{c}\text { R. } \\
\text { Personales }\end{array}$ & Cautela & $\begin{array}{c}\text { Origina- } \\
\text { lidad }\end{array}$ \\
\hline A20R cree que toda la gente es esencialmente honesta & 1 & 2 & 3 & 4 & 5 \\
B32R tiene mucha fe en la gente & 0.077 & 0.128 & 0.631 & -0.031 & 0.178 \\
B38R le simpatiza a toda la gente & 0.041 & 0.184 & 0.621 & 0.085 & -0.307 \\
D37R está lleno(a) de vigor y energía & 0.168 & -0.123 & 0.555 & 0.007 & 0.109 \\
C23R es muy cuidadoso(a) al tomar una decisión & 0.27 & 0.448 & -0.457 & 0.137 & 0.216 \\
A23R le gusta trabajar principalmente con ideas & 0.147 & 0.01 & -0.014 & 0.704 & 0.309 \\
D19R piensa mucho antes de tomar decisiones & 0.114 & 0.214 & 0.049 & -0.686 & -0.137 \\
A19R tiene ideas muy originales & 0.124 & -0.025 & 0.073 & 0.67 & -0.141 \\
C22R piensa las cosas con mucho cuidado antes de actuar & 0.229 & 0.273 & 0.075 & 0.503 & -0.034 \\
B34R es muy cauteloso(a) antes de actuar & 0.167 & 0.218 & -0.097 & -0.559 & 0.275 \\
D32R le agrada resolver problemas complicados & 0.238 & 0.221 & -0.136 & 0.439 & 0.036 \\
C25R es capaz de trabajar durante largos lapsos & 0.151 & 0.139 & -0.247 & -0.023 & 0.66 \\
D31R disfruta los problemas que requieren bastante reflexión & 0.214 & -0.284 & 0.094 & -0.174 & 0.488 \\
D38R no tiene un gran interés en adquirir conocimientos & -0.129 & -0.089 & -0.407 & 0.006 & -0.408 \\
C24R puede hacer mucho en poco tiempo & -0.171 & 0.15 & 0.194 & 0.048 & 0.578 \\
D26R tiene un gran sed de conocimientos & 0.304 & 0.266 & -0.111 & -0.064 & 0.328 \\
\hline
\end{tabular}

Nota: $\mathrm{n}=600$

Al calcular cinco factores fijos, se obtiene una mejor coherencia semántica de los factores con los constructos propuestos por Gordon (1972; 1994) dado que emergen los cuatro originales más un nuevo factor, que se denominó autocontrol emocional. Los reactivos que cargan en este nuevo factor se relacionan con el control de la impulsividad, el control de las emociones y la irritabilidad. El autocontrol emocional se diferenciaría de la escala de cautela en sus dimensiones emocional y cognoscitiva: los reactivos que cargan en cautela se caracterizan por un alto contenido en actividad cognoscitiva, toma de decisiones, previsión y planeación; mientras que los reactivos de autocontrol emocional se caracterizan por un control inhibitorio o regulatorio de la emoción.
La solución factorial obtenida arrojó un $\mathrm{KMO}$ de .77 y explica el $39.95 \%$ de la varianza total acumulada. Al revisar por cada componente, el primer factor de autocontrol emocional explicó el 10.96\% de la varianza, el segundo factor de vigor explicó el $7.85 \%$, el tercer factor de relaciones personales explicó el $7.39 \%$, el cuarto factor de cautela explicó el 7.24\%, y finalmente el quinto factor de originalidad explicó el $6.05 \%$ de la varianza.

De acuerdo a la solución factorial obtenida, se procedió a calcular los coeficientes de fiabilidad Alfa de Cronbach y los descriptivos de media y desviación estándar para las nuevas escalas. Dos de los coeficientes de fiabilidad son pobres para las escalas de Originalidad y Vigor; dos 
son cuestionables para Relaciones interpersonales y Cautela; y, finalmente, el coeficiente de la nueva escala de Autocontrol Emocional resultó ser aceptable según la clasificación de George y Mallerly (2003). Los resultados se detallan en la Tabla 8.

Tabla 8

Coeficientes Alfa de Cronbach de las Escalas Revisadas del IPG.

\begin{tabular}{lcccc}
\hline Escala & Elementos & $\alpha$ & M & DE \\
\hline $\begin{array}{l}\text { Autocontrol } \\
\text { Emocional }\end{array}$ & 11 & 0.74 & 15.04 & 3.92 \\
Vigor & 5 & 0.55 & 7.43 & 1.74 \\
$\begin{array}{l}\text { Relaciones } \\
\text { Personales }\end{array}$ & 5 & 0.61 & 4.48 & 2.07 \\
$\begin{array}{l}\text { Cautela } \\
\text { Originalidad }\end{array}$ & 6 & 0.68 & 7.12 & 2.28 \\
\hline
\end{tabular}

Nota: $n=600$

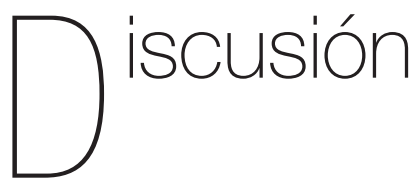

El objetivo general del presente estudio fue evaluar la validez y la confiabilidad factorial del P-IPG en una muestra de Bogotá - Colombia, para lo cual se realizaron diversos análisis estadísticos en una muestra de 600 participantes para estimar las propiedades psicométricas de las escalas que lo componen. Dado que la prueba en realidad está compuesta por dos sub-pruebas de desarrollo independiente se realizaron dos análisis separados tanto para el PPG como para el IPG. En general, los resultados de los dos estudios indican que la prueba conocida como P-IPG parece tener un comportamiento factorial distinto en población colombiana que en la población original ya que las estructuras factoriales no pudieron ser replicadas de manera satisfactoria en esta muestra.
Por lo anterior, fue requerido realizar una propuesta de solución factorial para el contexto específico en cada subprueba; esto permitió aproximarse de manera más acertada a la identificación de aquellos reactivos que realmente están midiendo los constructos de interés en esta muestra en particular. Aunque los reactivos identificados por el manual como solución factorial original fueron en parte diferentes a los reactivos identificados en la muestra de Bogotá (Colombia), efectivamente fue posible encontrar soluciones factoriales adecuadas que en general guardaron coherencia semántica con los constructos originales propuestos por Gordon (1972; 1994). Al respecto, y si bien los ítems identificados como críticos fueron distintos en la muestra estadounidense frente a la muestra colombiana, en general la coherencia semántica en el agrupamiento de los reactivos valida los constructos y las escalas estudiadas.

Se encontraron comportamientos factoriales específicos en la muestra, especialmente lo que atañe a la solución factorial de cinco factores para el IPG. Este aspecto es quizás uno de los hallazgos más importantes de la presente investigación dado que se trata de un resultado inesperado sugiriendo que, por lo menos en esta muestra, el IPG en realidad mide cinco constructos y no cuatro como lo postula Gordon (1972; 1994). Este comportamiento factorial diferencial sugiere que la prueba P-IPG posee una clave de puntuación específica para el contexto colombiano y que dichas claves deberían ser reformuladas para Colombia. Adicionalmente, es posible construir versiones abreviadas del P-IPG con las soluciones factoriales propuestas en la presente investigación; este aspecto es crítico para 
la investigación en el área de la personalidad que se conduzca en Colombia dado que las soluciones factoriales identifican claramente aquellos reactivos que realmente miden el constructo en este contexto y garantiza cierto grado de pureza factorial. En todo caso, contar con esta información es crucial para los investigadores que utilicen el P-IPG como medida de la personalidad en Colombia.

En conclusión, los resultados de la validez factorial indican que el P-IPG es una prueba válida para su uso en Colombia siendo posible obtener soluciones factoriales admisibles que respaldan las escalas propuestas por Gordon (1972; 1994) en la muestra estudiada. En las escalas efectivamente existen reactivos que se agrupan de acuerdo a estos factores propuestos por el autor, mostrando cargas factoriales superiores a 0.40. Las cargas factoriales se ajustan a los estándares internacionales para la identificación de factores al existir un considerable número de reactivos que superan cargas de 0.40 en el factor teóricamente esperado (Hernández \& González, 2006).

Según Cervone y Pervin (2009), el análisis factorial es de alta importancia en la teoría de los rasgos, ya que es la herramienta empleada para identificar las estructuras de la personalidad. Para la mayoría de teóricos de los rasgos, los factores que son identificados en estudios de análisis factorial son estructuras de la personalidad. En este mismo sentido, se utilizaron los análisis factoriales en la presente investigación, lo que permitió vincular los factores matemáticos identificados en la presente muestra con la teoría de los rasgos de la personalidad que sustenta el P-IPG como test psicológico.
En cuanto a la confiabilidad de las escalas completas del P-IPG, se hace necesario diferenciar entre las dos subpruebas. Cabe aclarar que el PPG tuvo, tanto en la estructura factorial como en la fiabilidad, un mejor comportamiento factorial que el IPG. El PPG muestra niveles de fiabilidad en el rango de .63 a .72 que tradicionalmente se consideran como aceptables. El IPG tuvo un comportamiento similar donde el coeficiente alfa de Cronbach osciló entre .60 y .68. Aunque tradicionalmente suele aceptarse un coeficiente de fiabilidad superior a .60, algunos autores consideran que los coeficientes adecuados se encuentran entre .70 y .90 (Campos \& Oviedo, 2008).

La confiabilidad de la prueba, según los resultados del presente estudio, difieren significativamente de lo presentado por Gordon (1972; 1994) en el manual de la prueba para muestras estadounidenses, ya que el rango de coeficientes alfa de Cronbach para el PPG se encuentra entre .85 a .88 y para el IPG entre .83 y .87, categorizados como buenos según la clasificación de George y Mallery (2003). Mientras que, en el presente estudio, utilizando la misma clasificación, la mayoría de los coeficientes de las escalas del P-IPG se ubicarían como cuestionables a excepción de la escala de responsabilidad (.72) que es aceptable. De todas maneras, no existe un acuerdo absoluto frente a los valores tolerables en los coeficientes de fiabilidad y la prueba puede usarse en este contexto ya que supera el valor mínimo aceptable de .60 de fiabilidad en todas sus escalas. Para resumir en una frase, el instrumento P-IPG resulto ser válido y confiable en una muestra colombiana. 
Dentro de las limitaciones se encuentra que, si bien el tamaño muestral es amplio y suficiente (soportado por los coeficientes KMO y Bartlett hallados en los segundos análisis) y que el muestreo fue por bola de nieve y no por sujetos disponibles, la muestra fue obtenida de forma no probabilística y de una sola parte del país, por lo que se sugiere estudios con mayores muestras y de diferentes zonas de Colombia. Debe igual señalarse que éste es un estudio inédito en el contexto colombiano, por lo que es necesario realizar investigaciones similares replicando estos hallazgos para corroborar estos resultados.

Para futuras investigaciones se hace relevante contrastar los presentes resultados respecto a validez y confiabilidad del P-IPG ya que se encontraron algunos hallazgos inesperados como los cuestionables coeficientes alfa de Cronbach de algunas de las escalas y la solución pentafactorial del IPG. Lo ideal sería la conducción de un estudio que considerase como técnica el análisis factorial confirmatorio de la estructura de la prueba PIPG. Se sugiere también mejorar el muestreo en lo posible probabilístico y, en todo caso, se hace necesaria la estandarización de la prueba para el contexto particular colombiano, adicional de un proceso de revisión de su validez convergente, con instrumentos similares o con teorías cercanas a la de los rasgos. La construcción de baremos específicos para Colombia se hace absolutamente necesaria dado que al diferir la estructura factorial de la original; lo más probable es que los baremos colombianos también difieran.

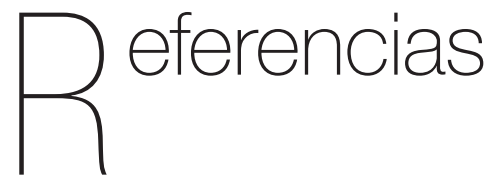

Anastasi, A., \& Urbina, S. (1998). Tests Psicológicos. 2da Edición. México: Prentice Hall.

Bravo del Toro, A., Espinosa, T., Mancilla, L. \& Tello, M. (2011). Rasgos de personalidad en pacientes con obesidad. Enseñanza e Investigación en Psicología, 16(1), 115-123.

Brown, G. (1980). Principios de la Medición en Psicología. México: Editorial Manual Moderno.

Campos, A. \& Oviedo, H. (2008). Propiedades Psicométricas de una Escala: la Consistencia Interna. Revista de Salud Pública, 10(5), 831-839.

Cervone, D. \& Pervin, L. (2009). Personalidad: teoría e investigación. México: Manual moderno.

George, D. \& Mallery, P. (2003). SPSS for Windows step by step: a simple guide and reference. 11.0 update ( $4^{\mathrm{a}}$ ed.). Boston, MA: Allyn \& Bacon.

González, C. (2009). Propiedades Psicométricas de la Escala de Desesperanza de Beck en una Muestra Bogotana. Psychologia: Avances de la Disciplina, 3(2), 17-30.

Gordon, L. (1972). Perfil - Inventario de Personalidad (P-IPG). México: Manual Moderno. 
Gordon, L. (1994). Perfil - Inventario de Personalidad (P-IPG). México: Manual Moderno.

Hernández, R. \& González, M. (2006). Estadística con SPSS y Metodología de la Investigación. México: Trillas.

Hernández, R., Fernández, C., \& Baptista, P. (2014). Metodología de la Investigación, quinta edición. México: McGraw Hill.

IBM Corp. Released 2013. IBM SPSS Statistics for Windows, Version 22.0. Armonk, NY: IBM Corp.

Kerlinger, F., \& Lee, H. (2002). Investigación del Comportamiento: Métodos de Investigación en Ciencias Sociales. México: Mc Graw Hill.

Martínez, H., Romero B., E. \& Trejo M., C. (1994). Perfil e Inventario de la Personalidad (trad., L. V. Gordon). México: Manual Moderno.

McCrae, R. \& Costa, P. (1999). A five-factor Theory of personality. En Pervin, L.A. \& John, O.P (eds) Handbook of Personality: Theory and research (139-153). New York: Guilford.
Montero, I. \& León, O. (2007). Guía para Nombrar los Estudios en Psicología. International Journal of Clinical and Health Psychology, 7(3), 867-862.

Peña, D. (2002). Análisis de datos multivariantes. Madrid: McGraw Hill.

Pérez, C. (2005). Técnicas Estadísticas con SPSS 12: Aplicaciones al análisis de datos. Madrid: Prentice Hall.

Rodríguez, C. M., Ávila, A., González, M., \& Heredia, Y. (2008). Perfil psicosocial y uso de las Tecnologías de la Información y la Comunicación de alumnos con promedios académicos altos y mínimos de la modalidad educativa presencial en un contexto mexicano. Revista Electrónica de Investigación Educativa, 10(2). Recuperado de http://redie.uabc.mx/vol10no2/ contenidorodriguezavila.html

Sierra, Y., Barrera, V., Gómez, L. P., Jiménez, C., \& Vélez, I. (2008). Adaptación y pilotaje del cuestionario sobre el análisis de los factores psicosociales presentes en los docentes universitarios. Cuadernos Hispanoamericanos de Psicología, 8(1), 29-44. 